\title{
Chancroid Coexisting with Vaginal Candidiasis: A Case Report
}

\author{
Raja Tina Anggrainy Dwi Putri*(D), Asnawi Madjid (D), Widya Widita (D), Nahda Yaumil(D) \\ Department of Dermatology and Venereology, Faculty of Medicine, Hasanuddin University, Makassar, Indonesia
}

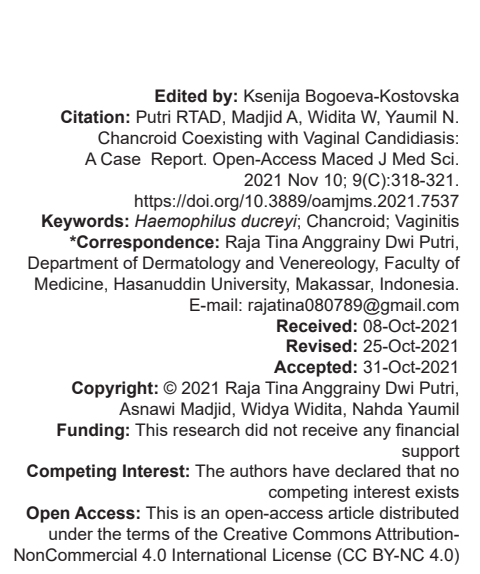

Abstract

BACKGROUND: Chancroid is a sexually transmitted disease caused by the Gram-negative bacteria, Haemophilus ducreyi. In endemic areas, the ratio of men to women is $3: 1$. Clinical symptoms of chancroid manifest as genital ulcers which are generally painful and tender with irregular edges. The base of the ulcer can be covered with yellow or gray necrotic purulent exudate, and often bleeds when rubbed. Vaginitis is an inflammation of the vagina and can be accompanied with symptoms such as vaginal discharge, complaints of itching, and pain. The most common cause is an imbalance of bacteria or normal flora in the vagina.

CASE REPORT: We report a case of chancroid in a 20 -year-old woman in conjunction with vaginal candidiasis that was later identified as Candida glabrata. The patient was then treated with a combination of oral antibiotics and antifungals, as well as normal saline dressing that then showed significant clinical improvements.

CONCLUSION: Chancroid is a sexually transmitted disease that is still prevalent especially in developing countries. Diagnosis of the disease is often made clinically and should be promptly treated to prevent further complications. Coexistence of chancroid with vaginal candidiasis is rare, and combination of antibiotics and antifungals, as well as normal saline dressing proved to be effective in treating the condition.

\section{Introduction}

Chancroid is a sexually transmitted disease characterized by painful genital ulcers with occasional inguinal lymphadenitis caused by Haemophilus ducreyi, Gram-negative bacilli bacteria [1].The United Nations Program on HIV and AIDS and the World Health Organization estimated that the annual prevalence of chancroid worldwide is at around 6 million cases, with the ratio of men compared to women at $3: 1$. The most prevalent age group is at 20-29 years [2]. However, there are currently no epidemiological data of chancroid available in Indonesia.

Infection of $H$. ducreyi occurs on the genital following epidermal microabrasion during sexual intercourse. It begins as an inflammatory papule that quickly develops into a painful ulcer within 1-2 days. In addition to the genital area, the lesions can also occur on the thighs and buttocks. Untreated chancroid can persist for months and can cause large ulcers as well as secondary bacterial infections [2].

Treatment options for chancroid include single-dose azithromycin $1 \mathrm{~g}$ oral, single-dose ceftriaxone $250 \mathrm{mg}$ intramuscular, or ciprofloxacin 500 mg twice daily oral. In pregnant patients, ceftriaxone is recommended, but azithromycin can still be given [3]. The patient education is essential, especially regarding treatment of sexual partners. In addition, patients are advised to not have sexual intercourse until they are completely healed [4]. This paper reports a case of chancroid in a 20-yearold woman in conjunction with vaginitis caused by Candida glabrata. The patient was treated with combination of antibiotics and systemic antifungals and showed clinical improvement.

\section{Case Report}

A 20-year-old woman came to the ER with the chief complaint of ulcers in the genital area 1 month prior. The initial complaint was the appearance of a dense painful erythematous nodule the size of green bean on the left genital area which then ruptured. Intense pain was felt especially when the lesions were touched or in contact with urine. However, dysuria was denied. In addition, there was no pruritus. The patient experienced no systemic symptoms such as fever. The patient had a history of recurrent vaginal discharge since the age of 15 which was clear in color, but in the past few months, the vaginal discharge had changed color to milky white and had an unpleasant odor. The patient denied any history of sexual intercourse or sexual abuse. 
Physical examination found that vital signs were within normal limits. Venereological examination showed multiple ulcers on the vulva with irregular undermined edges, purulent base that easily bleeds, and thick yellowish vaginal discharge. There were no enlarged lymph nodes (Figure 1a and b).

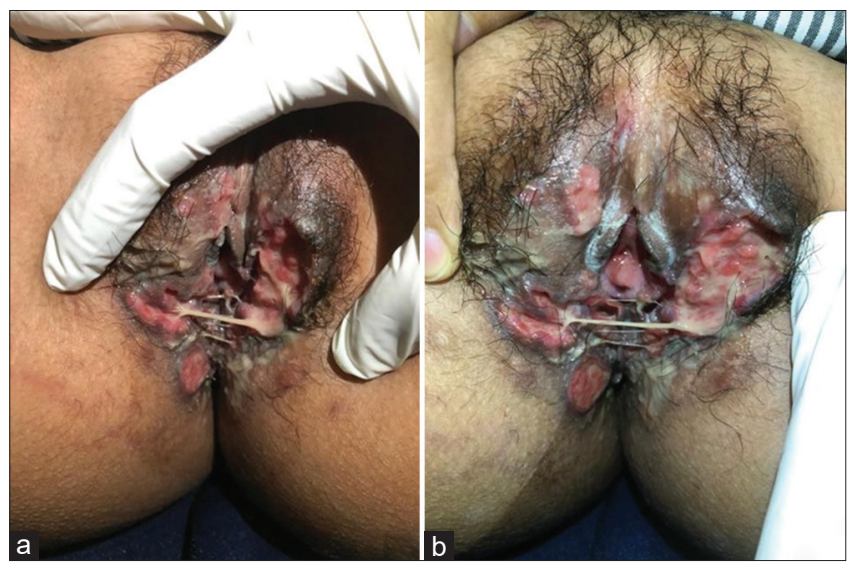

Figure 1: $(a$ and b) Multiple ulcers with irregular edges, resonant walls, dirty bottom, and mucopurulent vaginal discharge

Based on the initial history taking and physical examination, the patient was differentially diagnosed with chancroid, syphilis, and genital herpes. Laboratory tests of routine blood tests, immunoserology, Gram examination, $\mathrm{KOH}$, sniff test, and culture examination were performed. The results of the $\mathrm{KOH}$ examination and sniff test were negative. Routine blood tests were within normal limits. On immunoserological examination, VDRL/RPR, TPHA, and anti-HIV were also found to be non-reactive. Gram examination of vaginal discharge and pus revealed Gram-negative bacilli, suspected to be fastidious and accompanied by yeast (Figure 2a-c).

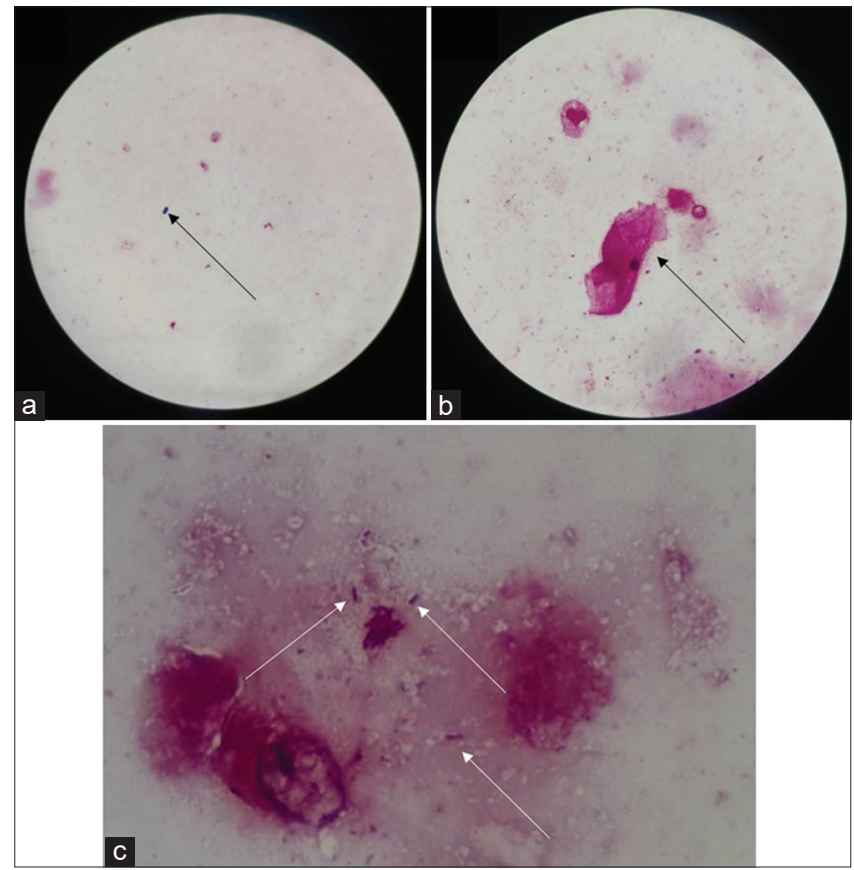

Figure 2: (a-c) Gram stain photo showed yeast +1 (a), epithelium +1 (b), and Gram-negative bacteria +1 (c)
After we have established laboratory examinations, a working diagnosis of chancroid was made. The patient was then treated with a combination of oral $500 \mathrm{mg}$ ciprofloxacin twice daily, analgesics, and application of normal saline dressing for 1-2 $\mathrm{h}$ on genital lesions. Six days after therapy, the patient felt reduction in pain and inflammation. No new lesions were observed. Vaginal discharge was still present (Figure 3). While waiting for the results of bacterial culture and sensitivity, the patient was discharged and asked to visit the outpatient clinic 1 week later.

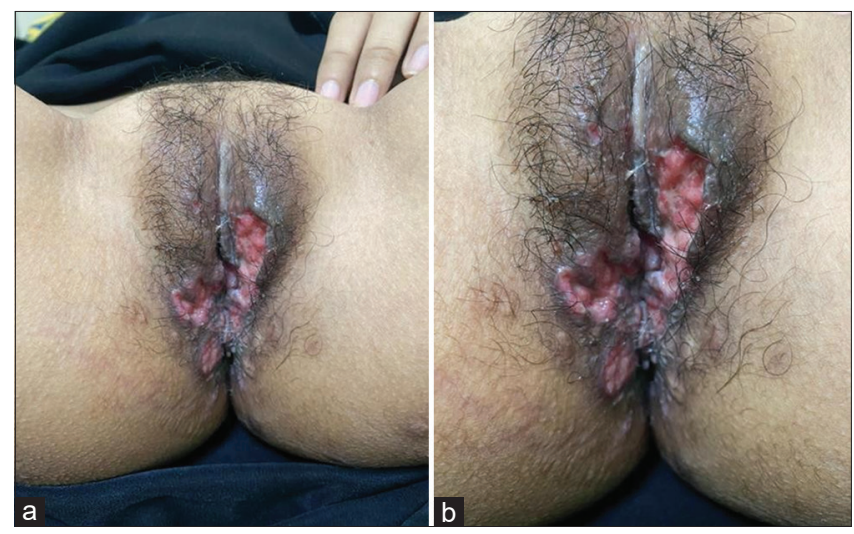

Figure 3: Clinical photo of the patient on day 6 showing multiple ulcers, irregular edges, resonant walls, dirty bottom. Fluorine albus (+) mucopurulent (a-b)

Fourteen days after the initial ER visit, culture result was Gram stained and found colonies of yeast that was later identified as C. glabrata using VITEK $2^{\mathrm{TM}}$ Technology, Biomérieux, Lyon (Figure 4). The patient was then prescribed additional systemic antifungal of $200 \mathrm{mg}$ ketoconazole daily for 14 days. After 2 weeks, the patient felt no vaginal discharge, leaving only superficial ulcers with irregular edges (Figure 5). Unfortunately, in our case, bacterial culture yielded negative result.

\section{Discussion}

Chancroid is a sexually transmitted disease caused by the rod-shaped Gram-negative bacteria $H$. ducreyi. Prevalence of the disease is poorly documented due to difficulties in microbial confirmation. A cohort study conducted in Papua New Guinea in 2014 showed evidence that $H$. ducreyi is a major cause of chronic skin ulceration. Similar studies reported similar findings in the Solomon Islands, Vanuatu, and Ghana [4]. This disease most often occurs in the youth population with a male-to-female ratio of $3: 1$, such as in this case of a 20 -year-old woman. Chancroid is characterized with painful genital ulcers and in $50 \%$ of cases accompanied by inguinal lymphadenitis. The incubation period is around 3-7 days, but in some cases, it can extend to 14 days, or longer especially in immunocompromised 


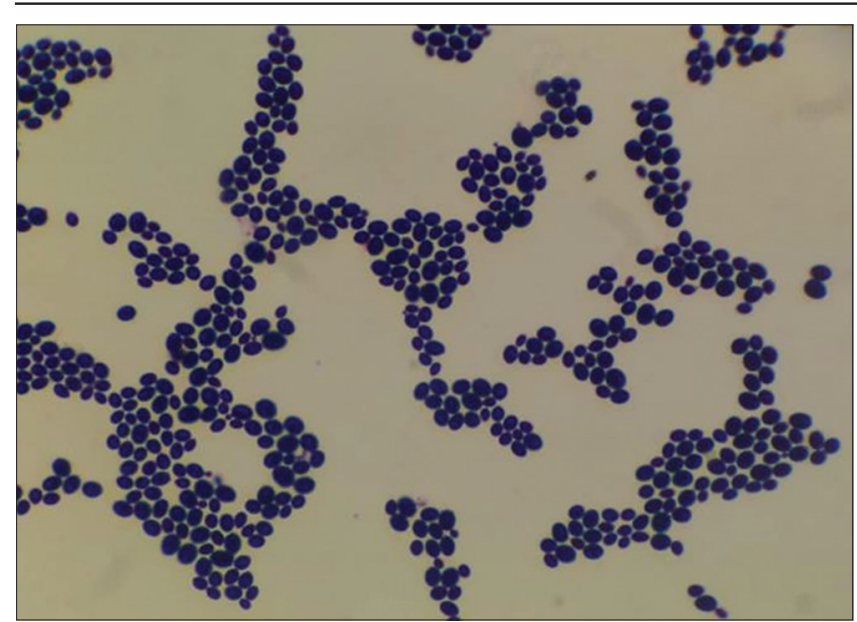

Figure 4: Gram stain of culture results and VITEK found colonies of yeast identified as Candida glabrata

patients. The disease has no prodromal symptoms. The infection process of $H$. ducreyi is thought to be in two ways, sexual transmission through skin-to-skin contact where microabrasion occurs during sexual intercourse [5] and through non-sexual transmission when pus-like fluid from the ulcer is transferred to other parts of the body or to other individuals [6].

After $H$. ducreyi enters the compromised skin either from abrasion, erosion, or excoriation due to trauma, or irritation related to poor personal hygiene, the host responds by sending polymorphonuclear

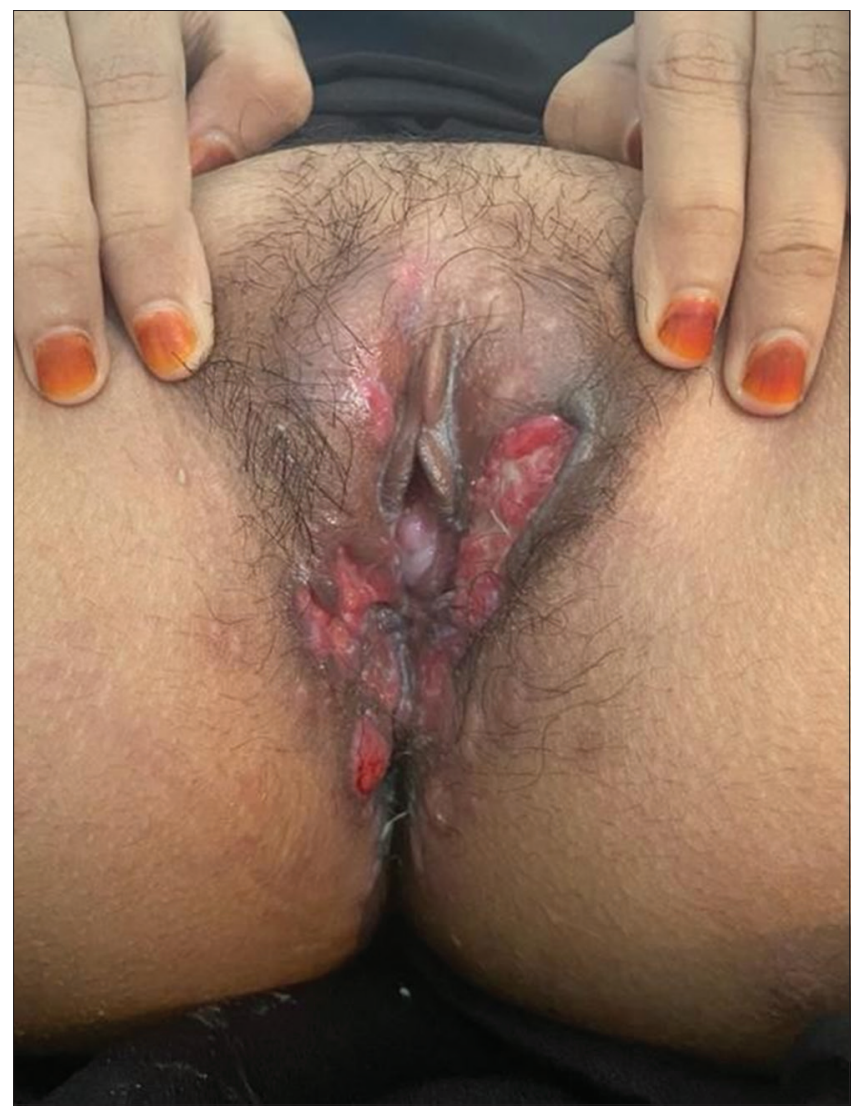

Figure 5: Clinical photo of the patient on day 14 showing multiple superficial ulcers with irregular edges leukocytes and macrophages leading to the development of initial lesions of papules and pustules that then evolve to ulcers. Recent studies on $H$. ducreyi suggest that pathogenesis focuses on how the organism inhibits phagocytosis and survives the activity of serum bactericidal components as well as antimicrobial peptides [7].

Ulcers may spread to the perineum, anus, scrotum, upper limbs, or lower abdomen because of autoinoculation [8]. The initial erythematous papules usually evolve into pustules within 4-7 days [9]. In the case, the patient experienced painful ulcers in the genital area 1 month prior admission to the hospital, where initially a solid red nodule the size of a seed appeared on the left genital area which then ruptured in 7 days. In addition, the patient also had a history of recurrent vaginal discharge in the past 5 years. The ulcer characteristics of chancroid are edges which are resonant, brittle, uneven, the skin, or mucosa surrounding the ulcer is erythematous. The base of the ulcer is lined with a grayish-yellow necrotic exudate and bleeds easily when the layer is removed [5]. The same thing was found in the patient.

Several examinations can be used to help identify $H$. ducreyi using samples such as pus and serum from genital lesions, such as microscopic examination, antigen detection, culture, nucleic acid detection, and serology tests [9]. Diagnosis can also be made clinically when characteristic symptoms are present [10]. This patient had three very painful ulcers on the labia majora. Gram examination of vaginal discharge revealed Gram-negative bacilli which were suspected to be fastidious and accompanied by yeast. Further culture found colonies of yeasts that were later identified as C. glabrata. Definitive diagnosis of $H$. ducreyi is difficult because it requires a positive culture or polymerase chain reaction (PCR) analysis. Unfortunately, at times, culture and PCR for $H$. ducreyi are not widely available or can yield negative result such as in this case. Therefore, in most occasions, diagnosis mostly relies on history taking and clinical findings [11].

Several antibiotic regimens are available to treat chancroid. First-line therapy includes singledose intramuscular $250 \mathrm{mg}$ ceftriaxone and singledose $1 \mathrm{~g}$ azithromycin. Second-line treatments include $500 \mathrm{mg}$ ciprofloxacin twice daily for 3 days and $500 \mathrm{mg}$ erythromycin 3-4x daily for 7 days [10], [11]. In this case, the patient responded well with oral ciprofloxacin with reduced inflammation and improvements on ulcers. However, as vaginal discharge kept on occurring, this prompted suspicion of a possible Candida infection. In some studies, C. glabrata infection can be difficult to treat due to the high numbers of resistance toward azole class antifungals. Usually, fluconazole can treat only half of the cases of vaginitis due to C. glabrata. However, in our case, the administration of ketoconazole $200 \mathrm{mg}$ daily for 7 days proved to be sufficient.

$H$. ducreyi and C. glabrata are rarely seen in otherwise healthy individuals and frequently occur in 
immunosuppressed patients, particularly AIDS. Acute HIV infection may mimic infectious disease because of non-specific prodromal signs, such as fatigue, headache, and weakness. Therefore, it is necessary to examine the serologic markers of the HIV [12]. However, the patient tested negative for HIV.

Patients with chancroid should be evaluated for other possible sexually transmitted diseases and should be done with an interval of around 3 months to exclude possible false negatives. The patient education to check and treat sexual partners is also important to prevent further recurrences in the future [4].

\section{Conclusion}

Chancroid is a sexually transmitted disease that is still prevalent especially in developing countries. Diagnosis of the disease is often made clinically and should be promptly treated to prevent further complications. Coexistence of chancroid with vaginal candidiasis is rare, and combination of antibiotics and antifungals, as well as normal saline dressing proved to be effective in treating the condition.

\section{References}

1. Spinola SM. Chancroid and Haemophilus ducreyi. In: Holmes KK, Sparling PF, Stamm WE, Piot P, Wasserheit JN, Corey L, editors. Sexually Transmitted Diseases. $4^{\text {th }}$ ed. Chicago: McGraw-Hill Education; 2007. p. 689-700.

2. González-Beiras $C$, Marks $M$, Chen $C Y$, Roberts $S$, Mitjà $O$. Epidemiology of Haemophilus ducreyi infections. Emerg Infect
Dis. 2016;22:1.

3. Spivey MI, Paschall RT, Ferrett R, Alexander R. Neisseria avoiding the jump to conclusions. J Child Sex Abuse. 2011;20:622-30.

4. Ulubay M, Keskin U, Fidan U, Alanbay I, Ozturk M, Ergun A Vulvar chancroid. Eur J Gen Med. 2015;12:180-2.

5. Hook EW, Handsfield HH. Gonococcal infections in the adult. In: Holmes KK, Sparling PF, Stamm WE, Piot P, Wasserheit JN, Corey L, editors. Sexually Transmitted Diseases. $4^{\text {th }}$ ed. Chicago: McGraw-Hill Education; 2007. p. 627-46.

6. Naveca FG, Sabidó M, de Almeida TA, Veras EA, Mejía MC Galban E, et al. Etiology of genital ulcer disease in a sexually transmitted infection reference center in Manaus, Brazilian Amazon. PLoS One. 2013;8(5):e63953. http://doi.org/10.1371/ journal.pone.0063953 PMid:23704961

7. Janowicz DM, Li W, Bauer ME. Host-pathogen interplay of Haemophilus ducreyi. Curr Opin Infect Dis. 2010;23(1):64-9. http://doi.org/10.1097/QCO.0b013e328334c0cb PMid:19918177

8. Sood S, Verma R, Mir SS, Agarwal M, Singh N, Kar HK et al. Nucleic acid amplification tests (NAATs) for gonorrhoea diagnosis in women: Experience of a tertiary care hospital in North India. Indian J Med Res. 2014;140(5):649-52.

PMid:25579147

9. Peel TN, Bhatti D, De Boer JC, Stratov I, Spelman DW Chronic cutaneous ulcers secondary to Haemophilus ducreyi infection. Med J Aust. 2010;192(6):348-50. http://doi. org/10.5694/j.1326-5377.2010.tb03537.x PMid:20230355

10. Kemp M, Christensen J, Lautenschlager S, Vall-Mayans $M$ Moi $\mathrm{H}$. European guideline for the management of chancroid, 2011. Int J STD AIDS. 2011;22(5):241-4. http://doi.org/10.1258/ ijsa.2010.010432 PMid:21571970

11. O'Farrell N, Lazaro N. UK National guideline for the management of Chancroid 2014. Int J STD AIDS. 2014;25(14):975-83. http:/l doi.org/10.1177/0956462414542988 PMid:25080286

12. Workowski KA, Bolan GA. Sexually transmitted diseases treatment guidelines, 2015. MMWR Recomm Rep. 2015;64(RR-03):1-137. 